\title{
NONEXISTENCE RESULTS OF SOLUTIONS TO SYSTEMS \\ OF SEMILINEAR DIFFERENTIAL INEQUALITIES \\ ON THE HEISENBERG GROUP
}

\author{
ABDALLAH EL HAMIDI AND MOKHTAR KIRANE
}

Received 25 December 2002

We establish nonexistence results to systems of differential inequalities on the $(2 N+1)$ Heisenberg group. The systems considered here are of the type $\left(\mathrm{ES}_{m}\right)$. These nonexistence results hold for $N$ less than critical exponents which depend on $p_{i}$ and $\gamma_{i}, 1 \leq i \leq m$. Our results improve the known estimates of the critical exponent.

\section{Introduction}

For the reader's convenience, we recall some background facts used here. The Heisenberg group $\mathbb{H}^{N}$, whose points will be denoted by $\eta=(x, y, \tau)$, is the Lie group $\left(\mathbb{R}^{2 N+1}, 0\right)$ with the group operation $\circ$ defined by

$$
\eta \circ \tilde{\eta}=(x+\tilde{x}, y+\tilde{y}, \tau+\tilde{\tau}+2(\langle x, \tilde{y}\rangle-\langle\tilde{x}, y\rangle)),
$$

where $\langle\cdot, \cdot\rangle$ is the usual inner product in $\mathbb{R}^{N}$. The Laplacian $\Delta_{\mathbb{H}}$ over $\mathbb{H}^{N}$ is obtained, from the vector fields $X_{i}=\partial_{x_{i}}+2 y_{i} \partial_{\tau}$ and $Y_{i}=\partial_{y_{i}}-2 x_{i} \partial_{\tau}$, by

$$
\Delta_{\mathbb{H}}=\sum_{i=1}^{N}\left(X_{i}^{2}+Y_{i}^{2}\right) \text {. }
$$

Observe that the vector field $T=\partial_{\tau}$ does not appear in (1.2). This fact makes us presume a "loss of derivative" in the variable $\tau$. The compensation comes from the relation

$$
\left[X_{i}, Y_{j}\right]=-4 T, \quad j, k \in\{1,2, \ldots, N\} .
$$

The relation (1.3) proves that $\mathbb{H}^{N}$ is a nilpotent Lie group of order 2. Incidently, (1.3) constitutes an abstract version of the canonical relations of commutation of Heisenberg between momentums and positions. Explicit computation gives the expression

$$
\Delta_{\mathbb{H}}=\sum_{i=1}^{N}\left(\frac{\partial^{2}}{\partial x_{i}^{2}}+\frac{\partial^{2}}{\partial y_{i}^{2}}+4 y_{i} \frac{\partial^{2}}{\partial x_{i} \partial \tau}-4 x_{i} \frac{\partial^{2}}{\partial y_{i} \partial \tau}+4\left(x_{i}^{2}+y_{i}^{2}\right) \frac{\partial^{2}}{\partial \tau^{2}}\right) .
$$


A natural group of dilatations on $\mathbb{H}^{N}$ is given by

$$
\delta_{\lambda}(\eta)=\left(\lambda x, \lambda y, \lambda^{2} \tau\right), \quad \lambda>0,
$$

whose Jacobian determinant is $\lambda^{Q}$, where

$$
Q=2 N+2
$$

is the homogeneous dimension of $\mathbb{H}^{N}$.

The operator $\Delta_{\mathbb{H}}$ is a degenerate elliptic operator. It is invariant with respect to the left translation of $\mathbb{H}^{N}$ and homogeneous with respect to the dilatations $\delta_{\lambda}$. More precisely, we have

$$
\begin{gathered}
\Delta_{\mathbb{H}}(u(\eta \circ \tilde{\eta}))=\left(\Delta_{\mathbb{H}} u\right)(\eta \circ \tilde{\eta}), \\
\Delta_{\mathbb{H}}\left(u \circ \delta_{\lambda}\right)=\lambda^{2}\left(\Delta_{\mathbb{H}} u\right) \circ \delta_{\lambda} \quad \forall(\eta, \tilde{\eta}) \in \mathbb{H}^{N} \times \mathbb{H}^{N} .
\end{gathered}
$$

It is natural to define a distance from $\eta$ to the origin by

$$
|\eta|_{\mathbb{H}}=\left(\tau^{2}+\sum_{i=1}^{N}\left(x_{i}^{2}+y_{i}^{2}\right)^{2}\right)^{1 / 4}
$$

In [7], Pohozaev and Véron gave another proof of the result of Birindelli et al. [1] concerning the nonexistence of weak solutions of the differential inequality

$$
\Delta_{\mathbb{H}}(a u)+|\eta|_{\mathbb{H}}^{\gamma}|v|^{p} \leq 0 \quad \text { in } \mathbb{H}^{N}
$$

for $\gamma>-2,1<p \leq(Q+\gamma) /(Q-2)$, and $a \in L^{\infty}\left(\mathbb{H}^{N}\right)$.

They then addressed the question of nonexistence of weak solutions of the system $\left(\mathrm{ES}_{2}\right)$ :

$$
-\Delta_{\mathbb{H}}\left(a_{1} u\right) \geq|\eta|_{\mathbb{H}}^{\gamma_{1}}|v|^{p_{1}}, \quad-\Delta_{\mathbb{H}}\left(a_{2} v\right) \geq|\eta|_{\mathbb{H}}^{\gamma_{2}}|u|^{p_{2}}
$$

where $a_{i}, i \in\{1,2\}$, are measurable and bounded functions defined on $\mathbb{H}^{N}$, and $p_{i}>1$ and $\gamma_{i}, i=1,2$, are real numbers. They showed that this system admits no solution defined in $\mathbb{H}^{N}$ whenever $\gamma_{i}>-2$ and $1<p_{i} \leq\left(Q+\gamma_{i}\right) /(Q-2), i=1,2$. The estimates on $p_{i}, i=1,2$, are obtained using Young's inequality and are not optimal. Using the Hölder inequality, we obtain better estimates on $p_{i}, 1 \leq i \leq m$. The same strategy is suitable to study the systems $\left(\mathrm{PS}_{m}\right)$ and $\left(\mathrm{HS}_{m}\right)$.

We also studied the following systems:

$$
\begin{gathered}
\left(\mathrm{PS}_{m}\right) \partial u_{i} / \partial t-\Delta_{\mathbb{H}}\left(a_{i} u_{i}\right) \geq|\eta|_{\mathbb{H}}^{\gamma_{i+1}}\left|u_{i+1}\right|^{p_{i+1}}, \eta \in \mathbb{H}^{N}, 1 \leq i \leq m, u_{m+1}=u_{1}, \\
\left(\mathrm{HS}_{m}\right) \partial^{2} u_{i} / \partial t^{2}-\Delta_{\mathbb{H}}\left(a_{i} u_{i}\right) \geq|\eta|_{\mathbb{H}}^{\gamma_{i+1}}\left|u_{i+1}\right|^{p_{i+1}}, \eta \in \mathbb{H}^{N}, 1 \leq i \leq m, u_{m+1}=u_{1},
\end{gathered}
$$

and showed the following results. 
Theorem 1.1. Assume that the initial data $u_{i}^{(0)} \in L^{1}\left(\mathbb{R}^{2 N+1}\right)$ and $\int u_{i}^{(0)}(\eta) d \eta \geq 0,1 \leq i \leq$ m. If

$$
Q \leq \max \left\{X_{1}, X_{2}, \ldots, X_{m}\right\}
$$

where the vector $\left(X_{1}, X_{2}, \ldots, X_{m}\right)^{T}$ is the solution of (3.1), then there is no nontrivial global weak solution $\left(u_{1}, \ldots, u_{m}\right)$ of the system $\left(P S_{m}\right)$.

Theorem 1.2. Assume that initial data (for the first derivatives of $u_{i}, 1 \leq i \leq m$ ) $u_{i}^{(1)} \in$ $L^{1}\left(\mathbb{R}^{2 N+1}\right)$ and $\int u_{i}^{(1)}(\eta) d \eta \geq 0,1 \leq i \leq m$. If

$$
Q \leq 1+\max \left\{X_{1}, X_{2}, \ldots, X_{m}\right\}
$$

where the vector $\left(X_{1}, X_{2}, \ldots, X_{m}\right)^{T}$ is the solution of (3.1), then there is no nontrivial global weak solution $\left(u_{1}, \ldots, u_{m}\right)$ of the system $\left(H S_{m}\right)$.

In [2], the first author and Obeid presented results for systems of evolution type with higher-order time derivatives. Their results are the generalized versions of our previous results (Theorems 1.1 and 1.2) on $\left(\mathrm{PS}_{m}\right)$ and $\left(\mathrm{HS}_{m}\right)$.

For interesting results on elliptic equations and systems, we refer to the recent papers of Kartsatos and Kurta [3], Kurta [4, 5], and Mitidieri and Pohozaev [6].

To render the presentation very clear, we start with the case of systems of two inequalities.

\section{Systems of two inequalities}

In this section, we treat the case $m=2$ and consider the system $\left(\mathrm{ES}_{2}\right)$.

We identify points in $\mathbb{H}^{N}$ with points in $\mathbb{R}^{2 N+1}$. We also recall that the Haar measure on $\mathbb{H}^{N}$ is identical to the Lebesgue measure $d \eta=d x d y d \tau$ on $\mathbb{R}^{2 N+1}=\mathbb{R}^{N} \times \mathbb{R}^{N} \times \mathbb{R}$. In the sequel, the integral $\int_{\mathbb{R}^{2 N+1}}$ will be simply denoted by $\int$; however, the measure of integration will be specified.

Definition 2.1. Let $a_{1}$ and $a_{2}$ be two bounded measurable functions on $\mathbb{R}^{2 N+1}$. A weak solution $(u, v)$ of the system $\left(\mathrm{ES}_{2}\right)$ on $\mathbb{R}^{2 N+1}$ is a pair of locally integrable functions $(u, v)$ such that

$$
u \in L_{\mathrm{loc}}^{p_{2}}\left(\mathbb{R}^{2 N+1},|\eta|_{\mathbb{H}}^{\gamma_{2}} d \eta\right), \quad v \in L_{\mathrm{loc}}^{p_{1}}\left(\mathbb{R}^{2 N+1},|\eta|_{\mathbb{H}}^{\gamma_{1}} d \eta\right)
$$

satisfying

$$
\begin{gathered}
\int_{\mathbb{R}^{2 N+1}}\left(a_{1} u \Delta_{\mathbb{H}} \varphi+|\eta|_{\mathbb{H}}^{\gamma_{1}}|v|^{p_{1}} \varphi\right) d \eta \leq 0, \\
\int_{\mathbb{R}^{2 N+1}}\left(a_{2} v \Delta_{\mathbb{H}} \varphi+|\eta|_{\mathbb{H}}^{\gamma_{2}}|u|^{p_{2}} \varphi\right) d \eta \leq 0
\end{gathered}
$$

for any nonnegative test function $\varphi \in C_{c}^{2}\left(\mathbb{R}^{2 N+1}\right)$. 
158 Nonexistence results to semilinear inequalities

Theorem 2.2. Assume that

$$
Q \leq Q_{e}^{*}=2+\frac{1}{p_{1} p_{2}-1} \max \left\{\left(\gamma_{1}+2\right)+p_{1}\left(\gamma_{2}+2\right) ; p_{2}\left(\gamma_{1}+2\right)+\left(\gamma_{2}+2\right)\right\} .
$$

Then there is no nontrivial weak solution $(u, v)$ of the system $\left(E S_{2}\right)$.

Proof. Let $\varphi_{R} \in \mathscr{D}\left(\mathbb{H}^{N}\right)$ be a nonnegative function such that

$$
\varphi_{R}(\eta)=\Phi^{\lambda}\left(\frac{\tau^{2}+|x|^{4}+|y|^{4}}{R^{4}}\right)
$$

where $\lambda \gg 1, R>0$, and $\Phi \in \mathscr{D}([0,+\infty[)$ is the "standard cutoff function"

$$
\Phi(r)=\left\{\begin{array}{ll}
1, & \text { if } 0 \leq r \leq 1, \\
0, & \text { if } r \geq 2
\end{array} \quad 0 \leq \Phi(r) \leq 1,\right.
$$

Note that $\operatorname{supp}\left(\varphi_{R}\right)$ is a subset of

$$
\Omega_{R}=\left\{\eta \equiv(x, y, \tau) \in \mathbb{H}^{N} ; 0 \leq \tau^{2}+|x|^{4}+|y|^{4} \leq 2 R^{4}\right\}
$$

and $\operatorname{supp}\left(\Delta_{\mathbb{H}} \varphi_{R}\right)$ is included in

$$
\mathscr{C}_{R}=\left\{\eta \equiv(x, y, \tau) \in \mathbb{H}^{N} ; R^{4} \leq \tau^{2}+|x|^{4}+|y|^{4} \leq 2 R^{4}\right\} .
$$

Let

$$
\rho=\frac{\tau^{2}+|x|^{4}+|y|^{4}}{R^{4}}
$$

then

$$
\begin{aligned}
\Delta_{\mathbb{H}} \varphi_{R}(\eta)= & \frac{4(N+4) \Phi^{\prime}(\rho)}{R^{4}} \lambda \Phi^{\lambda-1}(\rho)\left(|x|^{2}+|y|^{2}\right) \\
& +\frac{16 \Phi^{\prime \prime}(\rho)}{R^{8}} \lambda \Phi^{\lambda-1}(\rho) \\
& \times\left(\left(|x|^{6}+|y|^{6}\right)+\tau^{2}\left(|x|^{2}+|y|^{2}\right)+2 \tau\langle x, y\rangle\left(|x|^{2}-|y|^{2}\right)\right) \\
& +\frac{16 \Phi^{\prime 2}(\rho)}{R^{8}} \lambda(\lambda-1) \Phi^{\lambda-2}(\rho) \\
& \times\left(\left(|x|^{6}+|y|^{6}\right)+\frac{\tau^{2}}{4}\left(|x|^{2}+|y|^{2}\right)+2 \tau\langle x, y\rangle\left(|x|^{2}-|y|^{2}\right)\right) .
\end{aligned}
$$

It follows that there is a positive constant $C>0$, independent of $R$, such that

$$
\left|\Delta_{\mathbb{H}} \varphi_{R}(\eta)\right| \leq \frac{C}{R^{2}} \quad \forall \eta \in \Omega_{R}
$$


Let $(u, v)$ be a nontrivial weak solution of $\left(\mathrm{ES}_{2}\right)$. Using $(2.2)$ with $\varphi=\varphi_{R}$, one has

$$
\begin{aligned}
\int|\eta|_{\mathbb{H}}^{\gamma_{1}}|v|^{p_{1}} \varphi_{R} d \eta & \leq-\int a_{1} u \Delta_{\mathbb{H}} \varphi_{R} d \eta \\
& \leq \|\left. a_{1}\right|_{L^{\infty}} \int|u|\left|\Delta_{\mathbb{H}} \varphi_{R}\right| d \eta \\
& \leq\left.|| a_{1}\right|_{L^{\infty}}\left(\int|\eta|_{\mathbb{H}}^{\gamma_{2}}|u|^{p_{2}} \varphi_{R}\right)^{1 / p_{2}}\left(\int\left|\Delta_{\mathbb{H}} \varphi_{R}\right|^{p_{2}^{\prime}}\left(\varphi_{R}|\eta|_{\mathbb{H}}^{\gamma_{2}}\right)^{1-p_{2}^{\prime}}\right)^{1 / p_{2}^{\prime}} \\
\int|\eta|_{\mathbb{H}}^{\gamma_{2}}|u|^{p_{2}} \varphi_{R} d \eta & \leq-\int a_{2} v \Delta_{\mathbb{H}} \varphi_{R} d \eta \\
& \leq\left.|| a_{2}\right|_{L^{\infty}}\left(\int|\eta|_{\mathbb{H}}^{\gamma_{1}}|v|^{p_{1}} \varphi_{R}\right)^{1 / p_{1}}\left(\int\left|\Delta_{\mathbb{H}} \varphi_{R}\right|^{p_{1}^{\prime}}\left(\varphi_{R}|\eta|_{\mathbb{H}}^{\gamma_{1}}\right)^{1-p_{1}^{\prime}}\right)^{1 / p_{1}^{\prime}}
\end{aligned}
$$

thanks to the Hölder inequality. Setting

$$
I(R)=\int|\eta|_{\mathbb{H}}^{\gamma_{2}}|u|^{p_{2}} \varphi_{R} d \eta, \quad J(R)=\int|\eta|_{\mathbb{H}}^{\gamma_{1}}|v|^{p_{1}} \varphi_{R} d \eta,
$$

we have

$$
J(R) \leq C_{1} I(R)^{1 / p_{2}} \mathscr{A}_{p_{2}, \gamma_{2}}(R)^{1 / p_{2}^{\prime}},
$$

where

$$
\mathscr{A}_{p_{2}, \gamma_{2}}(R)=\int\left|\Delta_{\mathbb{H}} \varphi_{R}\right|^{p_{2}^{\prime}}\left(\varphi_{R}|\eta|_{\mathbb{H}}^{\gamma_{2}}\right)^{1-p_{2}^{\prime}} d \eta
$$

and $C_{1}$ is a positive constant independent of $R$. Similarly, we have

$$
I(R) \leq C_{2} J(R)^{1 / p_{1}} \mathscr{A}_{p_{1}, \gamma_{1}}(R)^{1 / p_{1}^{\prime}},
$$

where

$$
\mathscr{A}_{p_{1}, \gamma_{1}}(R)=\int\left|\Delta_{\mathbb{H}} \varphi_{R}\right|^{p_{1}^{\prime}}\left(\varphi_{R}|\eta|_{\mathbb{H}}^{\gamma_{1}}\right)^{1-p_{1}^{\prime}} d \eta
$$

and $C_{2}$ is a positive constant independent of $R$.

Note that for $\lambda$ sufficiently large, the integrals $\mathscr{A}_{p_{i}, \gamma_{i}}(R), i \in\{1,2\}$, are convergent. Indeed, in the expression $\mathscr{A}_{p_{i}, \gamma_{i}}(R), i \in\{1,2\}$, we have $|\eta|_{\mathbb{H}} \geq R^{4}$, and the exponent of $\varphi_{R}$ is positive for $\lambda$ large enough.

In order to estimate the integrals $\mathscr{A}_{p_{i}, \gamma_{i}}(R), i \in\{1,2\}$, we introduce the scaled variables

$$
\tilde{\tau}=R^{-2} \tau, \quad \tilde{x}=R^{-1} x, \quad \tilde{y}=R^{-1} y .
$$

Using the fact that $\operatorname{supp} \varphi_{R} \subset \Omega_{R}$, we conclude that

$$
\mathscr{A}_{p_{i}, \gamma_{i}}(R) \leq C R^{2 N+2-2 p_{i}^{\prime}+\gamma_{i}\left(1-p_{i}^{\prime}\right)}, \quad i \in\{1,2\} .
$$

Using (2.16) and (2.19) in (2.14), we obtain

$$
J(R)^{1-1 / p_{1} p_{2}} \leq C \mathscr{A}_{p_{1}, \gamma_{1}}(R)^{1 / p_{1}^{\prime} p_{2}} \mathscr{A}_{p_{2}, \gamma_{2}}(R)^{1 / p_{2}^{\prime}} \leq C R^{\sigma_{J}},
$$


160 Nonexistence results to semilinear inequalities

where

$$
\begin{aligned}
\sigma_{J} & =\frac{1}{p_{2}^{\prime}}\left(2 N+2-2 p_{2}+\gamma_{2}\left(1-p_{2}^{\prime}\right)\right)+\frac{1}{p_{1}^{\prime} p_{2}}\left(2 N+2-2 p_{1}+\gamma_{1}\left(1-p_{1}^{\prime}\right)\right) \\
& =Q\left(1-\frac{1}{p_{1} p_{2}}\right)-\frac{\left(2 p_{2}+2+\gamma_{2}\right) p_{1}+\gamma_{1}}{p_{1} p_{2}}
\end{aligned}
$$

Similarly, we have

$$
I(R)^{1-1 / p_{1} p_{2}} \leq C \mathscr{A}_{p_{1}, \gamma_{1}}(R)^{1 / p_{1}^{\prime}} \mathscr{A}_{p_{2}, \gamma_{2}}(R)^{1 / p_{1} p_{2}^{\prime}} \leq C R^{\sigma_{I}}
$$

where

$$
\sigma_{I}=Q\left(1-\frac{1}{p_{1} p_{2}}\right)-\frac{\left(2 p_{1}+2+\gamma_{1}\right) p_{2}+\gamma_{2}}{p_{1} p_{2}}
$$

Now, we require that $\sigma_{I} \leq 0$ or $\sigma_{J} \leq 0$, which is equivalent to

$$
\begin{aligned}
Q \leq Q_{e}^{*} & =\frac{1}{p_{1} p_{2}-1} \max \left\{p_{1}\left(2\left(p_{2}+1\right)+\gamma_{2}\right)+\gamma_{1} ; p_{2}\left(2\left(p_{1}+1\right)+\gamma_{1}\right)+\gamma_{2}\right\} \\
& =2+\frac{1}{p_{1} p_{2}-1} \max \left\{\left(\gamma_{1}+2\right)+p_{1}\left(\gamma_{2}+2\right) ; p_{2}\left(\gamma_{1}+2\right)+\left(\gamma_{2}+2\right)\right\}
\end{aligned}
$$

In this case, the integrals $I(R)$ and $J(R)$, increasing in $R$, are bounded uniformly with respect to $R$. Using the monotone convergence theorem, we deduce that $|\eta|_{\mathbb{H}}^{\gamma_{1}}|v|^{p_{1}}$ and $|\eta|_{\mathbb{H}}^{\gamma_{2}}|u|^{p_{2}}$ are in $L^{1}\left(\mathbb{R}^{2 N+1}\right)$. Note that instead of (2.11) we have, more precisely,

$$
\begin{aligned}
\int|\eta|_{\mathbb{H}}^{\gamma_{1}}|v|^{p_{1}} \varphi_{R} d \eta & \leq\left.|| a_{1}\right|_{L^{\infty}}\left(\int_{\mathscr{C}_{R}}|\eta|_{\mathbb{H}}^{\gamma_{2}}|u|^{p_{2}} \varphi_{R} d \eta\right)^{1 / p_{2}} \mathscr{A}_{p_{2}, \gamma_{2}}(R)^{1 / p_{2}^{\prime}} \\
& \leq C \int_{\mathscr{C}_{R}}|\eta|_{\mathbb{H}}^{\gamma_{2}}|u|^{p_{2}} \varphi_{R} d \eta
\end{aligned}
$$

Finally, using the dominated convergence theorem, we obtain that

$$
\lim _{R \rightarrow+\infty} \int_{\mathscr{C}_{R}}|\eta|_{\mathbb{H}}^{\gamma_{2}}|u|^{p_{2}} \varphi_{R} d \eta=0
$$

Hence,

$$
\int|\eta|_{\mathbb{H}}^{\gamma_{1}}|v|^{p_{1}} d \eta=0
$$

which implies that $v \equiv 0$ and $u \equiv 0$ via (2.12). This contradicts the fact that $(u, v)$ is a nontrivial weak solution of $\left(\mathrm{ES}_{2}\right)$, which achieves the proof.

Remark 2.3. The critical exponent $Q_{e}^{*}$ can be written as

$$
Q_{e}^{*}=2+\max \left\{X_{1}, X_{2}\right\},
$$


where the vector $\left(X_{1}, X_{2}\right)^{T}$ is the solution of the linear system

$$
\left(\begin{array}{cc}
-1 & p_{1} \\
p_{2} & -1
\end{array}\right)\left(\begin{array}{l}
X_{1} \\
X_{2}
\end{array}\right)=\left(\begin{array}{l}
\gamma_{1}+2 \\
\gamma_{2}+2
\end{array}\right)
$$

Comment 2.4. In their paper, Pohozaev and Véron [7] showed that if

$$
1<p_{j} \leq \frac{Q+\gamma_{j}}{Q-2}, \quad j \in\{1,2\}
$$

then the system $\left(\mathrm{ES}_{2}\right)$ has no nontrivial weak solution. The condition (2.30) is equivalent to

$$
Q \leq 2+\min \left\{\frac{\gamma_{1}+2}{p_{1}-1} ; \frac{\gamma_{2}+2}{p_{2}-1}\right\}
$$

Theorem 2.2 gives a better estimate of the exponent. Indeed,

$$
\frac{\left(\gamma_{1}+2\right)+p_{1}\left(\gamma_{2}+2\right)}{p_{1} p_{2}-1}-\frac{\gamma_{2}+2}{p_{2}-1}=-\frac{p_{2}\left(\gamma_{1}+2\right)+\left(\gamma_{2}+2\right)}{p_{1} p_{2}-1}+\frac{\gamma_{1}+2}{p_{1}-1},
$$

which implies that

$$
\max \left\{\frac{\left(\gamma_{1}+2\right)+p_{1}\left(\gamma_{2}+2\right)}{p_{1} p_{2}-1} ; \frac{p_{2}\left(\gamma_{1}+2\right)+\left(\gamma_{2}+2\right)}{p_{1} p_{2}-1}\right\} \geq \min \left\{\frac{\gamma_{1}+2}{p_{1}-1} ; \frac{\gamma_{2}+2}{p_{2}-1}\right\} .
$$

\section{Systems of $m$ semilinear inequalities}

In this section, we give generalizations of the last results to systems with $m$ inequalities, $m \in \mathbb{N}^{*}$.

Let $\left(X_{1}, X_{2}, \ldots, X_{m}\right)$ be the solution of the linear system

$$
\left(\begin{array}{cccccccc}
1 & -p_{1} & 0 & 0 & \cdots & 0 & 0 & 0 \\
0 & 1 & -p_{2} & 0 & \cdots & 0 & 0 & 0 \\
\vdots & \vdots & \vdots & \vdots & \cdots & \vdots & \vdots & \vdots \\
0 & 0 & 0 & 0 & \cdots & 0 & 1 & -p_{m-1} \\
-p_{m} & 0 & 0 & 0 & \cdots & 0 & 0 & 1
\end{array}\right)\left(\begin{array}{c}
X_{1} \\
X_{2} \\
\vdots \\
X_{m-1} \\
X_{m}
\end{array}\right)=\left(\begin{array}{c}
-\gamma_{1}-2 \\
-\gamma_{2}-2 \\
\vdots \\
-\gamma_{m-1}-2 \\
-\gamma_{m}-2
\end{array}\right)
$$

where $p_{i}>1$ and $\gamma_{i}$ are given real numbers, $i \in\{1,2, \ldots, m\}$.

Consider the system $\left(\mathrm{ES}_{m}\right)$ :

$$
-\Delta_{\mathbb{H}}\left(a_{i} u_{i}\right) \geq|\eta|_{\mathbb{H}}^{\gamma_{i+1}}\left|u_{i+1}\right|^{p_{i+1}}, \quad \eta \in \mathbb{H}^{N}, 1 \leq i \leq m, u_{m+1}=u_{1},
$$

where $p_{m+1}=p_{1}, \gamma_{m+1}=\gamma_{1}$. 
162 Nonexistence results to semilinear inequalities

Definition 3.1. Let $a_{i}, i \in\{1,2, \ldots, m\}$, be $m$ bounded measurable functions on $\mathbb{R}^{2 N+1}$. A weak solution $\left(u_{1}, \ldots, u_{m}\right)$ of the system $\left(\mathrm{ES}_{m}\right)$ on $\mathbb{R}^{2 N+1}$ is a vector of locally integrable functions $\left(u_{1}, \ldots, u_{m}\right)$ such that

$$
u_{i} \in L_{\mathrm{loc}}^{p_{i}}\left(\mathbb{R}^{2 N+1},|\eta|_{\mathbb{H}}^{\gamma_{i}} d \eta\right), \quad i \in\{1,2, \ldots, m\},
$$

satisfying

$$
\begin{gathered}
\int_{\mathbb{R}^{2 N+1}}\left(a_{i} u \Delta_{\mathbb{H}} \varphi+|\eta|_{\mathbb{H}}^{\gamma_{i+1}}\left|u_{i+1}\right|{ }^{p_{i+1}} \varphi\right) d \eta \leq 0, \quad i \in\{1,2, \ldots, m-1\}, \\
\int_{\mathbb{R}^{2 N+1}}\left(a_{m} u_{m} \Delta_{\mathbb{H}} \varphi+|\eta|_{\mathbb{H}}^{\gamma_{1}}|u|^{p_{1}} \varphi\right) d \eta \leq 0
\end{gathered}
$$

for any nonnegative test function $\varphi \in C_{c}^{2}\left(\mathbb{R}^{2 N+1}\right)$.

Theorem 3.2. If $Q \leq 2+\max \left\{X_{1}, X_{2}, \ldots, X_{m}\right\}$, then system $\left(E S_{m}\right)$ has no nontrivial solution. Proof. In order to simplify the proof, we treat only the case $m=3$; the general case can be established in the same manner.

Let $\left(u_{1}, u_{2}, u_{3}\right)$ be a nontrivial weak solution of $\left(\mathrm{ES}_{m}\right)$. The inequalities (3.4), with $\varphi=$ $\varphi_{R}$ defined by (2.4), imply that

$$
\begin{aligned}
& \int|\eta|_{\mathbb{H}}^{\gamma_{1}}\left|u_{1}\right|^{p_{1}} \varphi_{R} d \eta \\
& \quad \leq\left.|| a_{3}\right|_{L^{\infty}}\left(\int|\eta|_{\mathbb{H}}^{\gamma_{3}}\left|u_{3}\right|^{p_{3}} \varphi_{R}\right)^{1 / p_{3}}\left(\int\left|\Delta_{\mathbb{H}} \varphi_{R}\right|^{p_{3}^{\prime}}\left(\varphi_{R}|\eta|_{\mathbb{H}}^{\gamma_{3}}\right)^{1-p_{3}^{\prime}}\right)^{1 / p_{3}^{\prime}}, \\
& \int|\eta|_{\mathbb{H}}^{\gamma_{2}}\left|u_{2}\right|^{p_{2}} \varphi_{R} d \eta \\
& \quad \leq\left.|| a_{1}\right|_{L^{\infty}}\left(\int|\eta|_{\mathbb{H}}^{\gamma_{1}}\left|u_{1}\right|^{p_{1}} \varphi_{R}\right)^{1 / p_{1}}\left(\int\left|\Delta_{\mathbb{H}} \varphi_{R}\right|^{p_{1}^{\prime}}\left(\varphi_{R}|\eta|_{\mathbb{H}}^{\gamma_{1}}\right)^{1-p_{1}^{\prime}}\right)^{1 / p_{1}^{\prime}}, \\
& \int|\eta|_{\mathbb{H}}^{\gamma_{3}}\left|u_{3}\right|^{p_{3}} \varphi_{R} d \eta \\
& \quad \leq\left.|| a_{2}\right|_{L^{\infty}}\left(\int|\eta|_{\mathbb{H}}^{\gamma_{2}}\left|u_{2}\right|^{p_{2}} \varphi_{R}\right)^{1 / p_{2}}\left(\int\left|\Delta_{\mathbb{H}} \varphi_{R}\right|^{p_{2}^{\prime}}\left(\varphi_{R}|\eta|_{\mathbb{H}}^{\gamma_{2}}\right)^{1-p_{2}^{\prime}}\right)^{1 / p_{2}^{\prime}} .
\end{aligned}
$$

Let

$$
\begin{aligned}
I_{i}(R) & =\int|\eta|_{\mathbb{H}}^{\gamma_{i}}\left|u_{i}\right|^{p_{i}} \varphi_{R} d \eta, \quad 1 \leq i \leq 3, \\
\mathscr{A}_{i}(R) & =\int\left|\Delta_{\mathbb{H}} \varphi_{R}\right|^{p_{i}^{\prime}}\left(\varphi_{R}|\eta|_{\mathbb{H}}^{\gamma_{i}}\right)^{1-p_{i}^{\prime}}, \quad 1 \leq i \leq 3,
\end{aligned}
$$

then there is a positive constant $C$ such that

$$
I_{1} \leq C I_{3}^{1 / p_{3}} \mathscr{A}_{3}^{1 / p_{3}^{\prime}}, \quad I_{2} \leq C I_{1}^{1 / p_{1}} \mathscr{A}_{1}^{1 / p_{1}^{\prime}}, \quad I_{3} \leq C I_{2}^{1 / p_{2}} \mathscr{A}_{2}^{1 / p_{2}^{\prime}}
$$


Hence, the estimates

$$
\begin{aligned}
& I_{1}^{1-1 / p_{1} p_{2} p_{3}} \leq C \mathscr{A}_{1}^{1 / p_{1}^{\prime} p_{2} p_{3}} \mathscr{A}_{2}^{1 / p_{2}^{\prime} p_{3}} \mathscr{A}_{3}^{1 / p_{3}^{\prime}}, \\
& I_{2}^{1-1 / p_{1} p_{2} p_{3}} \leq C \mathscr{A}_{1}^{1 / p_{1}^{\prime}} \mathscr{A}_{2}^{1 / p_{1} p_{2}^{\prime} p_{3}} \mathscr{A}_{3}^{1 / p_{1} p_{3}^{\prime}}, \\
& I_{3}^{1-1 / p_{1} p_{2} p_{3}} \leq C \mathscr{A}_{1}^{1 / p_{1}^{\prime} p_{2}} \mathscr{A}_{2}^{1 / p_{2}^{\prime}} \mathscr{A}_{3}^{1 / p_{1} p_{2} p_{3}^{\prime}}
\end{aligned}
$$

hold true.

In order to estimate the expressions $I_{i}, 1 \leq i \leq 3$, we use the scaled variables (2.18) and obtain

$$
I_{i}^{1-1 / p_{1} p_{2} p_{3}} \leq C R^{\sigma_{i}}, \quad 1 \leq i \leq 3
$$

where

$$
\begin{aligned}
& \sigma_{1}=\left(1-\frac{1}{p_{1} p_{2} p_{3}}\right)\left(Q-2-\frac{\left(\gamma_{1}+2\right)+p_{1}\left(\gamma_{2}+2\right)+p_{1} p_{2}\left(\gamma_{3}+2\right)}{p_{1} p_{2} p_{3}-1}\right), \\
& \sigma_{2}=\left(1-\frac{1}{p_{1} p_{2} p_{3}}\right)\left(Q-2-\frac{p_{2} p_{3}\left(\gamma_{1}+2\right)+\left(\gamma_{2}+2\right)+p_{2}\left(\gamma_{3}+2\right)}{p_{1} p_{2} p_{3}-1}\right), \\
& \sigma_{3}=\left(1-\frac{1}{p_{1} p_{2} p_{3}}\right)\left(Q-2-\frac{p_{3}\left(\gamma_{1}+2\right)+p_{1} p_{3}\left(\gamma_{2}+2\right)+\left(\gamma_{3}+2\right)}{p_{1} p_{2} p_{3}-1}\right) .
\end{aligned}
$$

Now, we require that, at least, one of $\sigma_{i}, 1 \leq i \leq 3$, is less than zero, which is equivalent to $Q \leq 2+\max \left\{X_{1}, X_{2}, X_{3}\right\}$, where the vector $\left(X_{1}, X_{2}, X_{3}\right)^{T}$ is the solution of

$$
\left(\begin{array}{ccc}
1 & -p_{1} & 0 \\
0 & 1 & -p_{2} \\
-p_{3} & 0 & 1
\end{array}\right)\left(\begin{array}{l}
X_{1} \\
X_{2} \\
X_{3}
\end{array}\right)=\left(\begin{array}{l}
-\gamma_{1}-2 \\
-\gamma_{2}-2 \\
-\gamma_{3}-2
\end{array}\right)
$$

Following the arguments used in the proof of Theorem 2.2 , we conclude that $\left(u_{1}, u_{2}, u_{3}\right) \equiv$ $(0,0,0)$. This ends the proof by contradiction.

\section{References}

[1] I. Birindelli, I. Capuzzo-Dolcetta, and A. Cutrì, Liouville theorems for semilinear equations on the Heisenberg group, Ann. Inst. H. Poincaré Anal. Non Linéaire 14 (1997), no. 3, 295-308.

[2] A. El Hamidi and A. Obeid, Systems of semilinear higher-order evolution inequalities on the Heisenberg group, J. Math. Anal. Appl. 280 (2003), no. 1, 77-90.

[3] A. G. Kartsatos and V. V. Kurta, On a comparison principle and the critical Fujita exponents for solutions of semilinear parabolic inequalities, J. London Math. Soc. (2) 66 (2002), no. 2, 351-360.

[4] V. V. Kurta, On the absence of positive solutions of elliptic equations, Mat. Zametki 65 (1999), no. 4, 552-561 (Russian), translated in Math. Notes 65 (1999), no. 3-4, 462-469.

[5] - On the absence of positive solutions to semilinear elliptic equations, Tr. Mat. Inst. Steklova 227 (1999), no. 18, 162-169 (Russian), translated in Proc. Steklov Inst. Math. 227 (1999), no. $4,155-162$. 


\section{Nonexistence results to semilinear inequalities}

[6] E. Mitidieri and S. I. Pohozaev, A priori estimates and blow-up of solutions to nonlinear partial differential equations and inequalities, Proc. Steklov Inst. Math. 234 (2001), 362 (Russian).

[7] S. Pohozaev and L. Véron, Nonexistence results of solutions of semilinear differential inequalities on the Heisenberg group, Manuscripta Math. 102 (2000), no. 1, 85-99.

Abdallah El Hamidi: Laboratoire de Mathématiques, Université de La Rochelle, avenue Michel Crépeau, 17000 La Rochelle Cedex 9, France

E-mail address: aelhamid@univ-lr.fr

Mokhtar Kirane: Laboratoire de Mathématiques, Université de La Rochelle, avenue Michel Crépeau, 17000 La Rochelle Cedex 9, France

E-mail address: mkirane@univ-lr.fr 


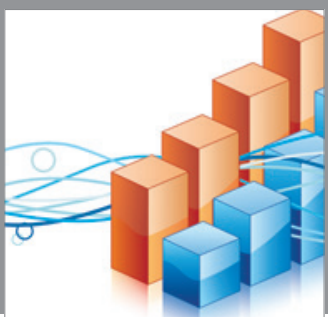

Advances in

Operations Research

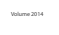

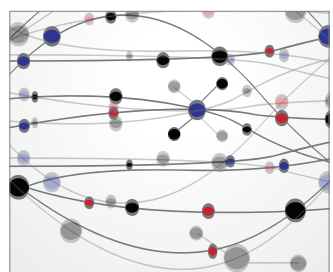

\section{The Scientific} World Journal
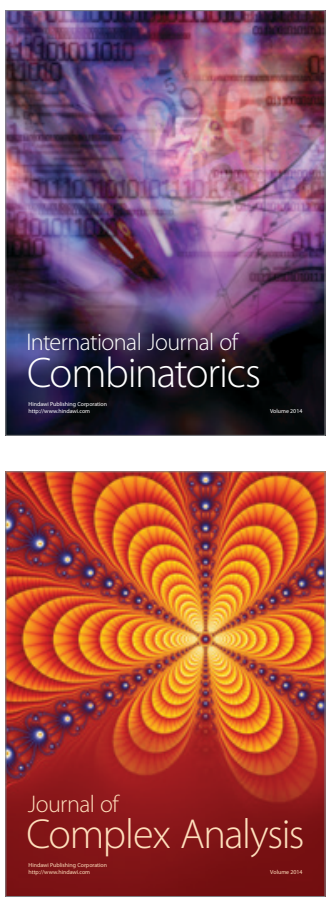

International Journal of

Mathematics and

Mathematical

Sciences
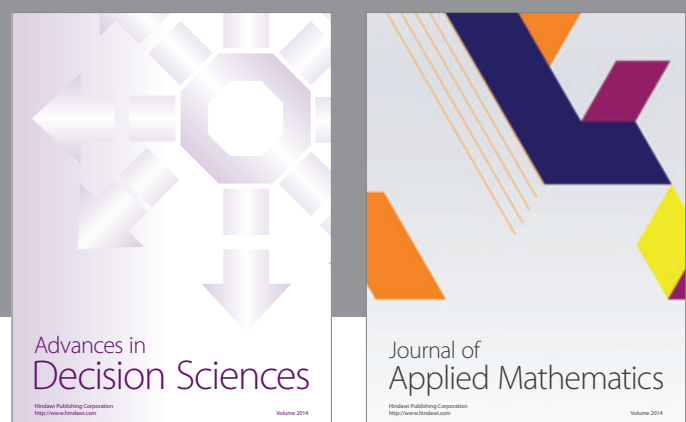

Journal of

Applied Mathematics
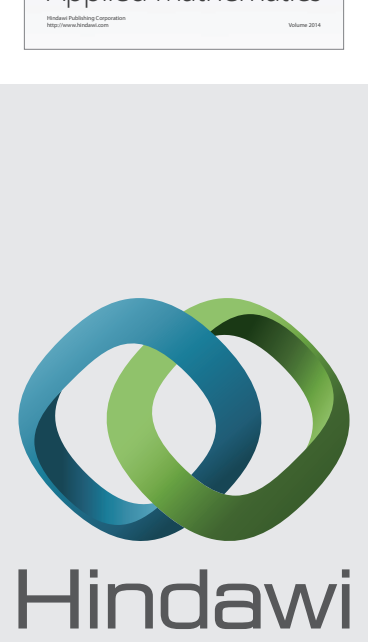

Submit your manuscripts at http://www.hindawi.com
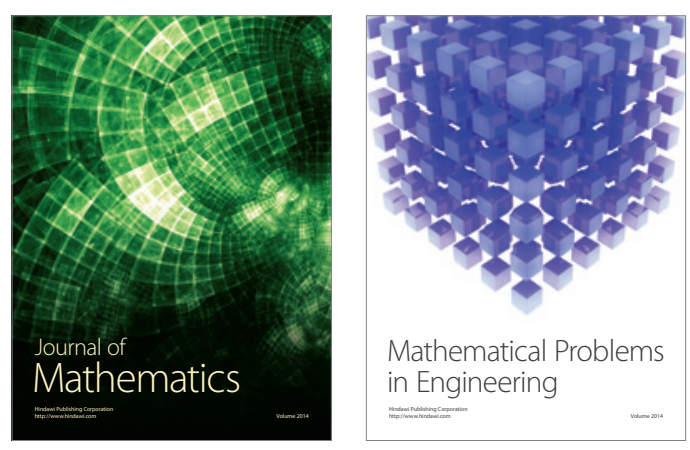

Mathematical Problems in Engineering
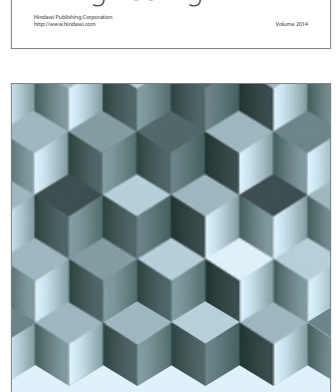

Journal of

Function Spaces
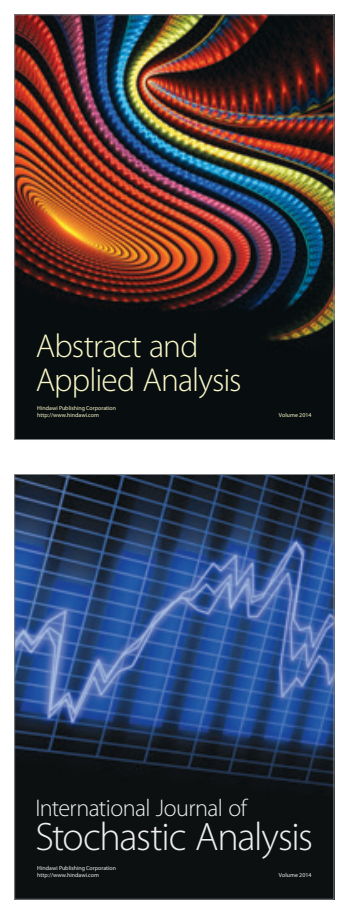

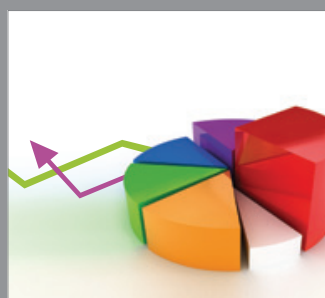

ournal of

Probability and Statistics

Promensencen
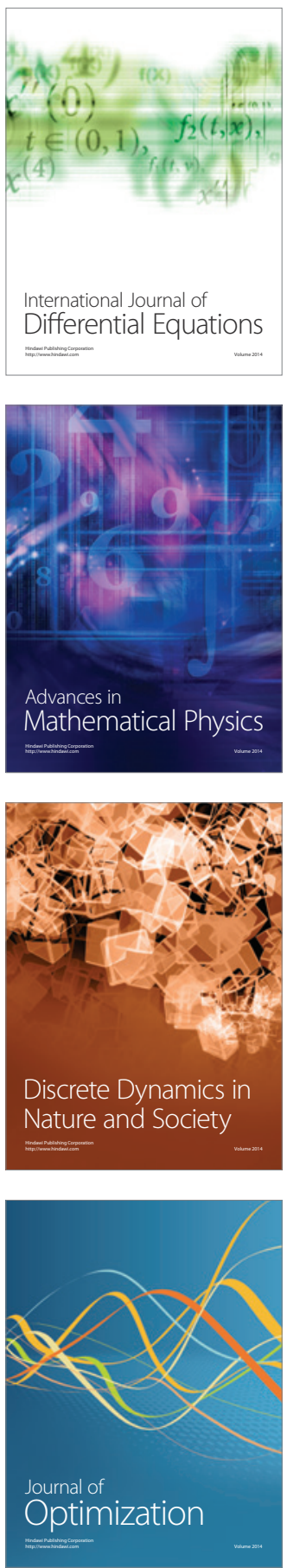